\title{
Small Dense Low-Density Lipoprotein Cholesterol is a Potential Marker for Predicting Laser Treatment for Retinopathy in Diabetic Patients
}

\author{
Atsuko Nakayama ${ }^{1}$ Hiroyuki Morita ${ }^{1}$ Tatsuyuki Sato ${ }^{1}$ Takuya Kawahara ${ }^{2}$ Norifumi Takeda ${ }^{1}$ Satoshi Kato ${ }^{3}$, \\ Hiroshi Itoh ${ }^{4}$ and Issei Komuro ${ }^{1}$ \\ Atsuko Nakayama and Hiroyuki Morita contributed equally to this work. \\ ${ }^{1}$ Department of Cardiovascular Medicine, Graduate School of Medicine, The University of Tokyo, Tokyo, Japan. \\ ${ }^{2}$ Clinical Research Promotion Center, The University of Tokyo, Tokyo, Japan. \\ ${ }^{3}$ Department of Ophthalmology, Graduate School of Medicine, The University of Tokyo, Tokyo, Japan. \\ ${ }^{4}$ Department of Endocrinology, Metabolism and Nephrology, Keio University School of Medicine, Tokyo, Japan.
}

Aim: We explored the superiority of small dense low-density lipoprotein cholesterol (sdLDL-C) as a marker for predicting not only the occurrence of cardiovascular $(\mathrm{CV})$ events but also the need for laser treatment in patients with hypercholesterolemia and diabetic retinopathy.

Methods: We performed a sub-analysis of the intEnsive statin therapy for hyper-cholesteroleMic Patients with diAbetic retinopaTHY (EMPATHY) study $(n=5042)$, in which patients were assigned randomly to intensive or standard statin therapy targeting low-density lipoprotein cholesterol $<70 \mathrm{mg} / \mathrm{dl}$ or $100-120 \mathrm{mg} / \mathrm{dl}$. Using the survival analysis, the risks for $\mathrm{CV}$ events and the need for laser treatment were evaluated according to the lipids one year after registration.

Results: The patients were $63 \pm 11$ years old. LDL-C and sdLDL-C levels were $98 \pm 25$ and $32 \pm 14 \mathrm{mg} / \mathrm{dl}$, respectively, one year after registration. The sdLDL-C level had a strong positive correlation with apolipoprotein $B$ level ( $r=0.83$ at registration). SdLDL-C was a sensitive marker for predicting $C V$ events when comparing among the quartiles according to sdLDL-C levels (hazard ratios: HR for quartiles 1-4 were 1.0, 1.4, 1.6, and 2.5, respectively; $p$ for trend $<0.01)$. Also, sdLDL-C was a sensitive marker for predicting the need for laser treatment among lipids (log rank, $p=0.009$ ), especially in patients with elderly ( $\geqq 65 \mathrm{yrs}$ ) and obesity (BMI $\geqq 25$ $\left.\mathrm{kg} / \mathrm{m}^{2}\right)$.

Conclusions: SdLDL-C is a sensitive target marker to predict cardiovascular events as well as the need for laser treatment in patients with hypercholesterolemia and diabetic retinopathy.

Trial registration: UMIN000003486, www.umin.ac.jp/ctr/.

Key words: Small dense low-density lipoprotein cholesterol, Diabetic retinopathy, Statin

\section{Introduction}

Low-density lipoprotein cholesterol (LDL-C) is a useful lipid marker for predicting cardiovascular $(\mathrm{CV})$ events $^{1,2)}$. Lipid-lowering therapy targeting LDL-C $<$ $70 \mathrm{mg} / \mathrm{dl}$ is recommended for patients with CV diseases ${ }^{3,4}$. Lipid markers other than LDL-C, such as total cholesterol (TC), high-density lipoprotein cholesterol (HDL-C), triglyceride (TG), triglyceriderich lipoprotein cholesterol (TRL-C), apolipoprotein A1 (ApoA1), and apolipoprotein B (ApoB), are also potential markers for predicting $\mathrm{CV}$ events ${ }^{1,2,5,6,7)}$.

Address for correspondence: Atsuko Nakayama, Department of Cardiovascular Medicine, Graduate School of Medicine, The University of Tokyo, 7-3-1 Hongo, Bunkyo-ku, Tokyo 113-8655, JAPAN E-mail: st7089-fki@umin.ac.jp

Received: February 28, 2021 Accepted for publication: March 31, 2021

Copyright@2021 Japan Atherosclerosis Society

This article is distributed under the terms of the latest version of CC BY-NC-SA defined by the Creative Commons Attribution License. 
One study reported that $A p o B / A 1$ is superior to LDL-C as a marker for predicting CV events ${ }^{2)}$. LDL-C is known to be subdivided into granular fractions, among which small dense LDL-C (sdLDL-C) with a smaller particle size has recently attracted attention as a lipid marker that may be more sensitive for predicting CV events than $\mathrm{LDL}-\mathrm{C}^{8)}$. LDL-C with a smaller particle size is more easily oxidized and permeable to the cell wall ${ }^{9)}$, promoting atherosclerotic changes more than LDL-C with a larger particle size, so-called large buoyant LDL-C (lbLDL-C). Several studies, including the Atherosclerosis Risk in Communities (ARIC) study, have reported sdLDL-C as a more sensitive predictive marker of CV events as compared with LDL-C ${ }^{8)}$.

Diabetic retinopathy as a common microvascular complication of diabetes is a primary cause of vision loss among adults leading to 0.8 million blindness and 3.7 million visual impairment in $2010^{10)}$. As timely treatment for diabetic retinopathy is known to reduce the risk of vision loss by $98 \%{ }^{11}$, predictive markers for the progress in diabetic retinopathy should be identified. Considering that diabetic retinopathy is caused by microvascular damage in retina, lipids which are known to have harmful effects on the vascular structure and function should be a predictive marker for the progress in diabetic retinopathy. Whether any lipid could be a powerful marker for the progress in diabetic retinopathy, is still inconclusive. Indeed, a randomized controlled trial in diabetic patients showed that treatment with fibrate reduced the need for laser treatment for diabetic retinopathy ${ }^{12}$. In this study, however, the mechanism of this effect did not seem to be related to lipid profile.

\section{Aim}

Here, we confirmed the previous evidence that sdLDL-C predicts the occurrence of CV events and investigated whether sdLDL-C predicts the need for laser treatment more sensitively than other lipid markers in patients with hypercholesterolemia and diabetic retinopathy using the dataset from the intEnsive statin therapy for hyper-cholesteroleMic Patients with diAbetic retinopaTHY (EMPATHY) study ${ }^{13)}$.

\section{Methods}

The EMPATHY study $(n=5042)$ was performed from 2010 to 2013, to examine whether intensive lipid-lowering therapy was superior to standard therapy in reducing the incidence of primary endpoints (i.e., CV events including cardiac, cerebral, renal, and vascular events, or CV-associated death) in patients with hypercholesterolemia and diabetic retinopathy, but no history of coronary artery disease. In this multicenter, prospective, randomized, openlabel, blinded endpoint (PROBE) study, patients were randomly assigned to intensive statin therapy targeting LDL-C $<70 \mathrm{mg} / \mathrm{dl}$, or standard statin therapy targeting LDL-C 100-120 mg/dl ${ }^{13)}$. The EMPATHY study showed only a tendency toward fewer CV events in the intensive therapy group as compared with the standard therapy group because the difference in LDL-C levels between the two groups one year after registration was smaller $(20 \mathrm{mg} / \mathrm{dl})$ than expected at planning the study.

Informed consent was obtained from each patient and the study was conducted under the Declaration of Helsinki and Japanese ethical guidelines for clinical studies. The protocol was reviewed and approved by the human research committee of each participating center (in total, 774 institutions).

\section{Study Population}

From the study population of the EMPATHY study $(n=5042)$, we excluded patients whose blood samples could not be obtained one year after registration $(n=453)$ (Fig. 1). After this exclusion, first, the correlation between lipid profile and the risk for CV events in 4589 patients was evaluated to confirm the previous evidence on the predictors for $\mathrm{CV}$ events. Next, patients with history of treatment for retinopathy including laser treatment $(n=1424)$ or without routine ophthalmologic check-up until 3 years after registration $(n=2189)$ were excluded. In the remaining 976 patients with routine ophthalmologic check-up after registration, we evaluated the correlation between lipid profile and the need for laser treatment for diabetic retinopathy.

\section{Outcomes}

The primary endpoints of this study included the occurrence of $\mathrm{CV}$ events and the need for laser treatment for diabetic retinopathy. $\mathrm{CV}$ events were defined as cardiac, cerebral, renal, and vascular events, or $\mathrm{CV}$-associated death ${ }^{13)}$. Cardiac events were defined as myocardial infarction or unstable angina requiring unscheduled hospitalization, or coronary revascularization. Cerebral events were defined as cerebral infarction or cerebral revascularization. Renal events were defined as initiation of chronic dialysis or an increase in serum creatinine levels by at least 2 -fold (and $>1.5 \mathrm{mg} / \mathrm{dl}$ ). Vascular events were defined as aortic disease, aortic dissection, mesenteric artery thrombosis, severe lower limb ischemia, revascularization, or finger/lower limb amputation

\section{Advance Publication Journal of Atherosclerosis and Thrombosis}




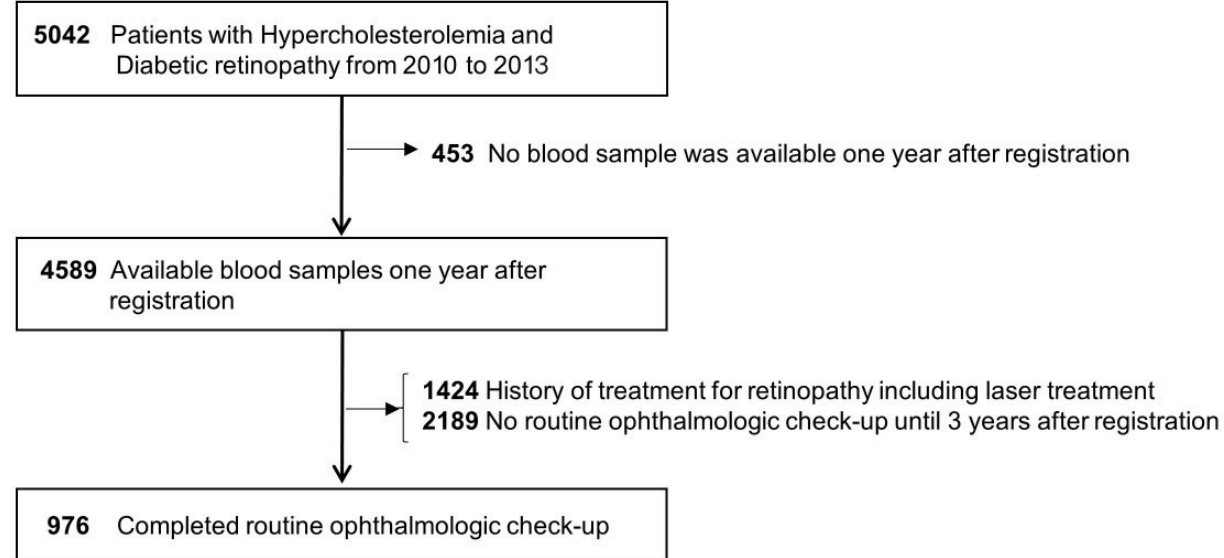

Fig. 1. Flowchart of patient enrollment

Patients whose blood samples were not available one year after registration were excluded from this study and the cardiovascular events were analyzed in remaining 4589 patients. Patients with the history of laser treatment for retinopathy and patients without routine ophthalmologic check-up until 3 years after registration were excluded from the analysis of the need for laser treatment.

caused by arteriosclerosis obliterans. Laser treatment for diabetic retinopathy was performed by ophthalmologists according to the guidelines for diabetic retinopathy ${ }^{14)}$.

\section{Experimental Design and Laboratory Methods}

In the EMPATHY study, blood samples were collected at each clinic from all patients at registration, and every year after registration, for analysis of lipid profile: TC, LDL-C, HDL-C, TG, ApoA1, ApoB, and sdLDL-C. TC levels were measured using the chemical oxygen demand method. LDL-C, HDL-C, and sdLDL-C levels were measured with direct homogenous assays using detergents (LDL-EX, HDL-EX, sdLDL-EX, Denka Seiken, Tokyo, Japan). TG levels were examined by the enzymatic assay of glycerol-3-phosphate oxidase. Apolipoprotein levels were measured using the turbidimetric immunoassay. All assays were performed at SRL (Tokyo, Japan). TRL-C was calculated as TC minus HDL-C minus LDL-C ${ }^{5}$.

\section{Management of Diabetic Retinopathy}

In the EMPATHY study, the retinal photographs of all participants were obtained at registration, indexing the stage of diabetic retinopathy according to the Early Treatment Diabetic Retinopathy Study (ETDRS) criteria ${ }^{15)}$. Routine ophthalmologic checkups were scheduled at registration and every year after registration. If the progression of diabetic retinopathy was suspected in the study period, additional ophthalmoscopy was performed according to demand. The information on the laser treatment for diabetic retinopathy was obtained from medical records in each institution. The laser treatment for macular edema was not regarded as the endpoint in the present study.

\section{Statistical Analyses}

Using SPSS ver. 22 (IBM, Chicago, IL, USA), the changes of lipids $0,1,2,3,4$ and 5 years after registration were evaluated by the paired or unpaired t-test. After the Kolmogorov-Smirnov Goodness-of-fit test, Pearson's correlation coefficient ( $\mathrm{r}$ ) was calculated to assess the degree of association between two variables in the parametric tests. The Spearman rank correlation coefficient was used to examine the degree of association between two variables in the nonparametric tests.

Hazard ratios (HRs) and survival curves were analyzed to compare the risk for CV events, death, and the need for laser treatment for retinopathy. To minimize the influence of lipid changing-term after registration, we set one year after registration as the landmark starting point to compare the outcomes among the groups and participants with events occurring $<1$ year after registration being excluded from the analysis. Covariates at registration, including age, gender, hypertension, and current or previous smoking, were adjusted in the Cox hazard analysis. For continuous variables, HRs of the Cox proportional analyses were estimated per one standard deviation increase ${ }^{16)}$. The Kaplan-Meier analysis and Cox hazard analysis were used to estimate survival curves of quartiles according to levels of LDL-C, TG, TRL-C, and sdLDL-C.

Since the higher prevalence of dyslipidemia including the elevated sdLDL-C level was reported in

\section{Advance Publication Journal of Atherosclerosis and Thrombosis}


Table 1. Baseline Characteristics at registration

\begin{tabular}{|c|c|c|}
\hline & Analysis on CV events $(n=4589)$ & Analysis on laser treatment $(n=976)$ \\
\hline Age (yrs) & $63 \pm 11$ & $63 \pm 14$ \\
\hline Male, $n(\%)$ & $2182(48)$ & $467(48)$ \\
\hline Body mass index $\left(\mathrm{kg} / \mathrm{m}^{2}\right)$ & $26 \pm 4$ & $26 \pm 4$ \\
\hline Systolic blood pressure (mmHg) & $135 \pm 16$ & $136 \pm 17$ \\
\hline Diastolic blood pressure (mmHg) & $75 \pm 11$ & $75 \pm 11$ \\
\hline Hypertension, $n(\%)$ & $3259(71)$ & $717(73)$ \\
\hline \multicolumn{3}{|l|}{ Smoking, $n(\%)$} \\
\hline Current & $842(18)$ & $165(17)$ \\
\hline Past & $993(22)$ & $225(23)$ \\
\hline No & $2754(60)$ & $586(60)$ \\
\hline Family history of CAD, $n(\%)$ & $590(13)$ & $144 \quad(15)$ \\
\hline$\beta$ blocker, $n(\%)$ & $272(6)$ & $55(6)$ \\
\hline ACEI, $n(\%)$ & $235(5)$ & $55(6)$ \\
\hline $\mathrm{ARB}, n(\%)$ & $2240(49)$ & $510(52)$ \\
\hline Intensive statin therapy, $n(\%)$ & $2272(50)$ & $500(51)$ \\
\hline $\mathrm{Hb}(\mathrm{g} / \mathrm{dl})$ & $14 \pm 2$ & $14 \pm 2$ \\
\hline Creatinine (mg/dl) & $0.8 \pm 0.3$ & $0.7 \pm 0.2$ \\
\hline $\mathrm{BNP}(\mathrm{pg} / \mathrm{ml})$ & $26 \pm 41$ & $27 \pm 47$ \\
\hline CRP (mg/dl) & $1.5 \pm 5.8$ & $1.4 \pm 4.5$ \\
\hline HbA1c (\%) & $7.2 \pm 1.2$ & $7.3 \pm 1.2$ \\
\hline \multicolumn{3}{|l|}{ Diabetic retinopathy status, $n(\%)$} \\
\hline Simple retinopathy & $3053(67)$ & $691(71)$ \\
\hline Preproliferative retinopathy & $840(18)$ & $193(20)$ \\
\hline Proliferative retinopathy & $664(14)$ & $92(9)$ \\
\hline Others ${ }^{\S}$ & $32(1)$ & $0(0)$ \\
\hline History of treatment for retinopathy & $1424(31)$ & $0(0)$ \\
\hline
\end{tabular}

diabetic nephropathy ${ }^{17)}$, the association between sdLDL-C level and the risk of the need for laser treatment for retinopathy was calculated after adjustment for the existence of nephropathy (defined as urine albumin to creatinine ratio $\geqq 30 \mathrm{mg} / \mathrm{gCr}$ and/ or estimated glomerular filtration rate $($ eGFR) $<30$ $\mathrm{ml} / \mathrm{min} / 1.73 \mathrm{~m}^{2}$ ).

\section{Results}

\section{Correlations among Lipids}

The baseline characteristics of patients are shown in Table 1. The average age in the total study population was 63 years old and $48 \%$ of them were male and the level of HbAlc was $7.2 \pm 1.2 \%$. Follow-up period was $3.2 \pm 0.9$ years. The changes of all lipids, except for ApoA1, were significantly changed one year after registration, and after that, lipid profile were not significantly changed except LDL-C and TRL-C at 2 years after registration, ApoA1 at 3 years after registration, and sdLDL-C at 2 and 5 years after registration (Table 2). Close correlations between sdLDL-C and other lipids, were observed at registration and one year after registration (Table 3). At registration, both of LDL-C and $A p o B$ were significantly correlated with sdLDL-C, and correlation between ApoB and sdLDL-C $(r=0.83, p<0.001)$ was greater as compared with that between LDL-C and sdLDL-C ( $\mathrm{r}=0.64, p<0.001)$ (Fig. 2).

\section{Survival Analysis}

Next, we analyzed the risk of variables for $\mathrm{CV}$ events (Fig. 3A). Male, higher systolic blood pressure, current or past smoker, lower level of hemoglobin, and higher levels of creatinine, brain natriuretic peptide (BNP), C-reactive protein, and $\mathrm{HbAlc}$ were significantly associated with the risk for $\mathrm{CV}$ events. The levels of all lipids at registration were not significantly related to the risk for $\mathrm{CV}$ events (data not shown). However, higher levels of TC, LDL-C, TG, 
Table 2. The longitudinal change of Lipids

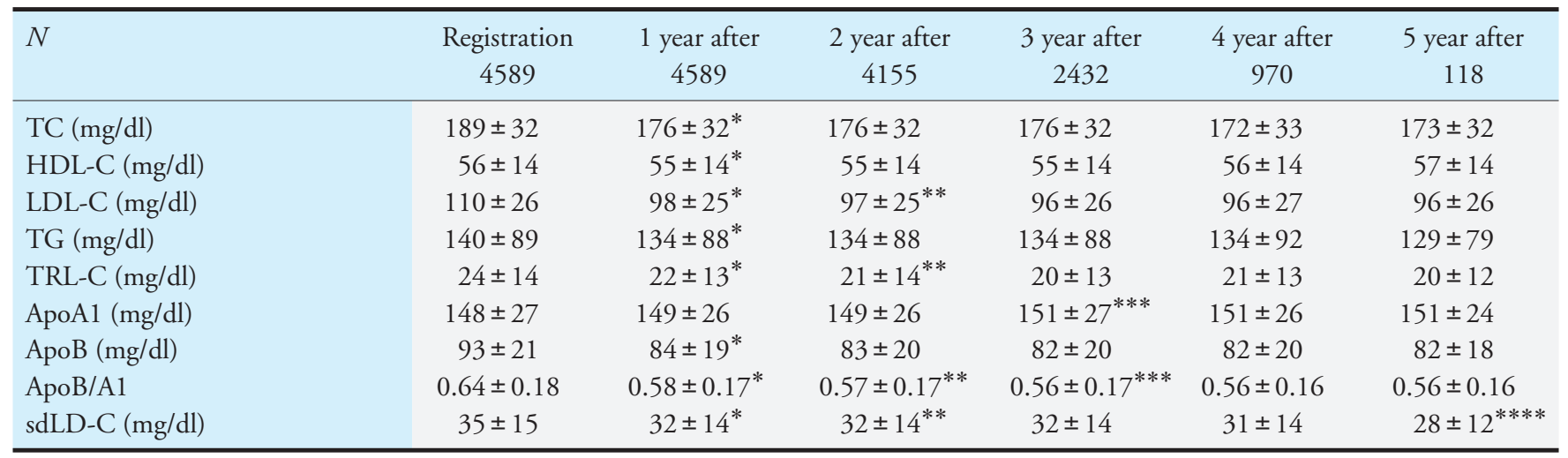

The levels of lipids were compared using the $t$ test. ${ }^{*} p<0.05$ between patients at registration and one year after registration. ${ }^{* *} p<0.05$ between patients with 1 year and 2 years after registration. ${ }^{* *} p<0.05$ between patients with 2 years and 3 years after registration. ${ }^{* * * *} p<0.05$ between patients with 4 years and 5 years after registration .TC: total cholesterol, HDL-C: high-density lipoprotein cholesterol, LDL-C: low-density lipoprotein cholesterol, TG: triglyceride, TRL-C: triglyceride-rich lipoprotein cholesterol, Apo A1: apolipoprotein A1, Apo B: apolipoprotein B, sdLDL-C: small dense LDL-C.

Table 3. Correlation between small dense LDL cholesterol and other biomarkers

\begin{tabular}{|c|c|c|}
\hline$n=4589$ & correlation coefficient & $p$ \\
\hline \multicolumn{3}{|l|}{ at registration } \\
\hline Age (yrs) & -0.14 & $<0.001$ \\
\hline $\mathrm{Hb}(\mathrm{g} / \mathrm{dl})$ & 0.21 & $<0.001$ \\
\hline Creatinine $(\mathrm{mg} / \mathrm{dl})$ & 0.00 & 0.81 \\
\hline $\mathrm{BNP}(\mathrm{pg} / \mathrm{ml})$ & -0.09 & $<0.001$ \\
\hline CRP (mg/dl) & -0.02 & 0.23 \\
\hline HbA1c (\%) & 0.20 & $<0.001$ \\
\hline \multicolumn{3}{|l|}{ Lipid at registration } \\
\hline $\mathrm{TC}(\mathrm{mg} / \mathrm{dl})$ & 0.66 & $<0.001$ \\
\hline HDL-C (mg/dl) & -0.16 & $<0.001$ \\
\hline LDL-C (mg/dl) & 0.64 & $<0.001$ \\
\hline $\mathrm{TG}(\mathrm{mg} / \mathrm{dl})$ & 0.58 & $<0.001$ \\
\hline TRL-C (mg/dl) & 0.53 & $<0.001$ \\
\hline ApoA1 (mg/dl) & 0.04 & $<0.01$ \\
\hline ApoB (mg/dl) & 0.83 & $<0.001$ \\
\hline ApoB/A1 & 0.63 & $<0.001$ \\
\hline \multicolumn{3}{|l|}{ Lipid after one year } \\
\hline $\mathrm{TC}(\mathrm{mg} / \mathrm{dl})$ & 0.67 & $<0.001$ \\
\hline HDL-C (mg/dl) & -0.16 & $<0.001$ \\
\hline LDL-C (mg/dl) & 0.67 & $<0.001$ \\
\hline $\mathrm{TG}(\mathrm{mg} / \mathrm{dl})$ & 0.58 & $<0.001$ \\
\hline TRL-C (mg/dl) & 0.49 & $<0.001$ \\
\hline ApoA1 (mg/dl) & 0.05 & $<0.01$ \\
\hline ApoB (mg/dl) & 0.84 & $<0.001$ \\
\hline ApoB/A1 & 0.64 & $<0.001$ \\
\hline
\end{tabular}

Hb: hemoglobin, BNP: brain natriuretic peptide, CRP: C-reactive protein, TC: total cholesterol, HDL C high density lipoprotein cholesterol, LDL C low density lipoprotein cholesterol, TG: triglyceride, TRL C: triglyceride-rich lipoprotein cholesterol, Apo A1: apolipoprotein A1, Apo B: apolipoprotein B 
A

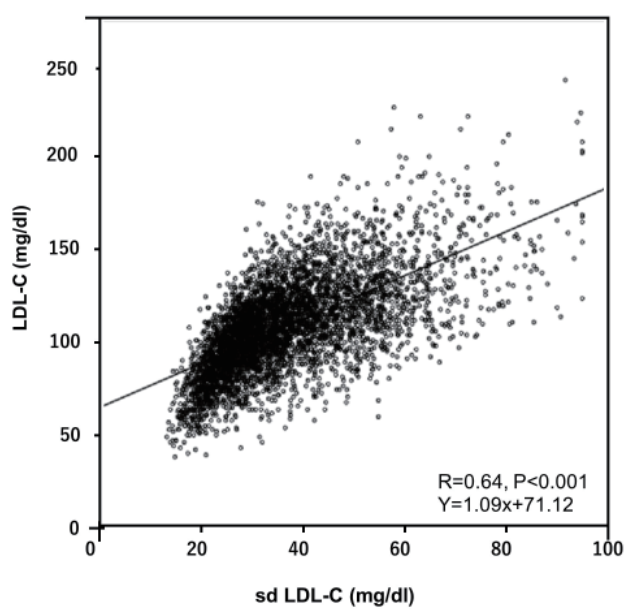

B

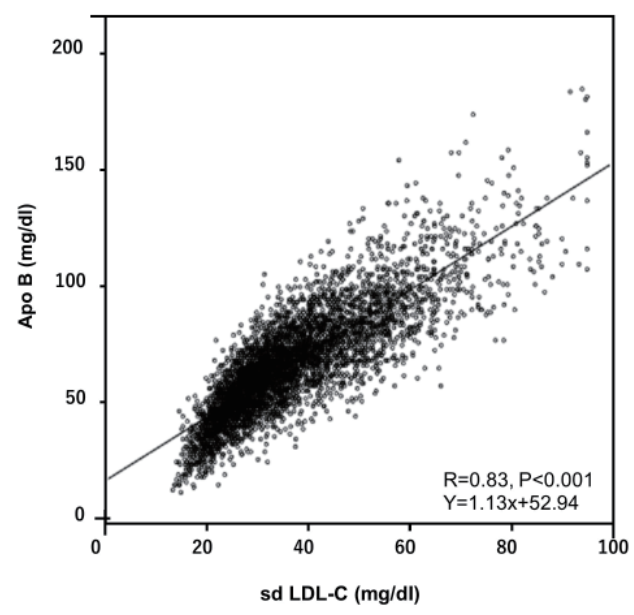

Fig. 2. Correlation between sdLDL-C and LDL-C or Apo B

A: The scatter diagrams of the correlation between LDL-C and sdLDL-C at registration. B: The scatter diagrams of the correlation between Apo B and sdLDL-C at registration. LDL-C: low-density lipoprotein cholesterol, sdLDL-C: small dense low-density lipoprotein cholesterol, Apo B: apolipoprotein B.

A

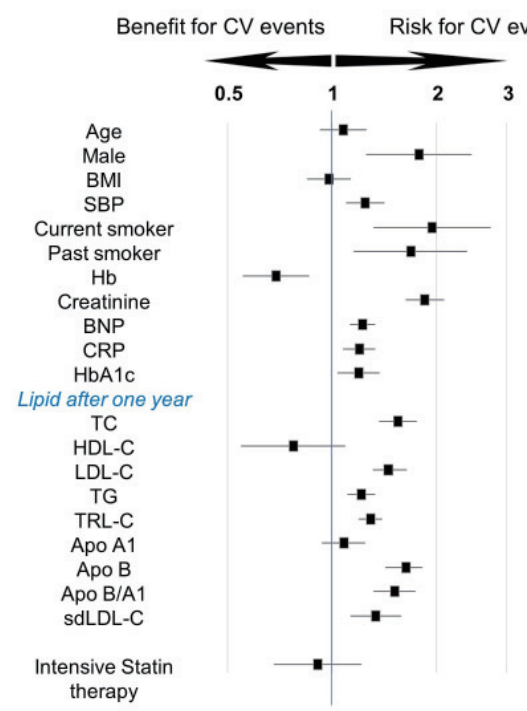

B

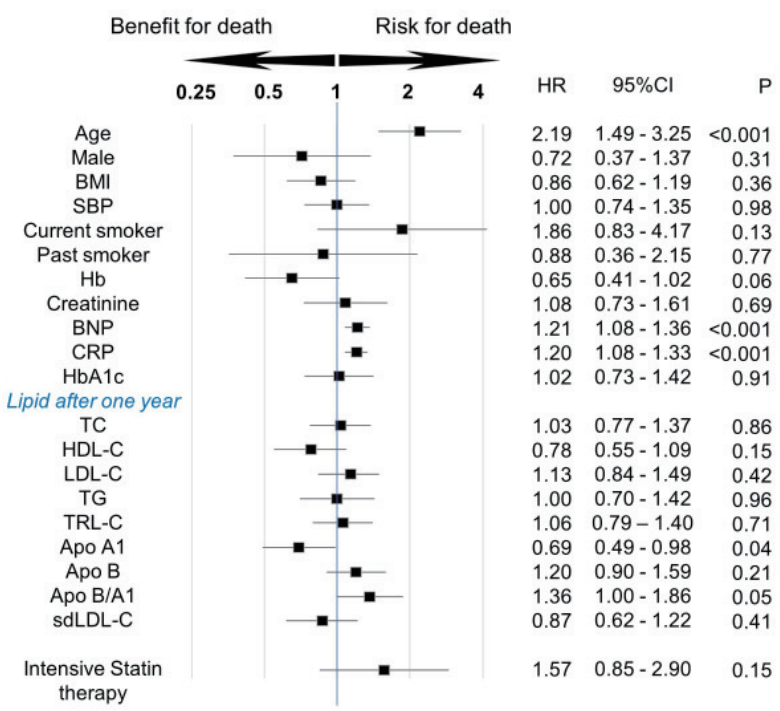

Fig. 3. Hazard ratios of each parameter for $\mathrm{CV}$ events and death

Hazard ratios were estimated per one standard deviation increase by a Cox proportional analysis of the risks for CV events (A) and all death (B), after adjusting for covariates including age, gender, hypertension, and current or previous smoking at registration. Hb: hemoglobin, BNP: brain natriuretic peptide, CRP: C-reactive protein, TC: total cholesterol, HDL-C: high density lipoprotein cholesterol, LDL-C: low density lipoprotein cholesterol, TG: triglyceride, TRL-C: triglyceride-rich lipoprotein cholesterol, ApoA1: apolipoprotein A1, Apo B: apolipoprotein B, sdLDL-C: small dense low-density lipoprotein cholesterol, CV: cardiovascular.

TRL-C, ApoB and sdLDL-C at one year after registration were significantly associated with the risk for CV events (HR 1.56, 95\% CI 1.37-1.77; HR 1.45 , 95\% CI 1.31-1.64; HR 1.19, 95\% CI 1.09 1.30; HR 1.29, 95\% CI 1.20-1.40; HR 1.63, 95\% CI 1.43-1.82; HR 1.34, 95\% CI 1.13-1.58, respectively).
While older age and higher levels of BNP and C-reactive protein were significantly associated with risk for all death, none of the lipid markers except for ApoA1 at one year after registration were related to the risk for death (Fig. 3B).

Comparisons among the quartiles (Q) according

\section{Advance Publication Journal of Atherosclerosis and Thrombosis}




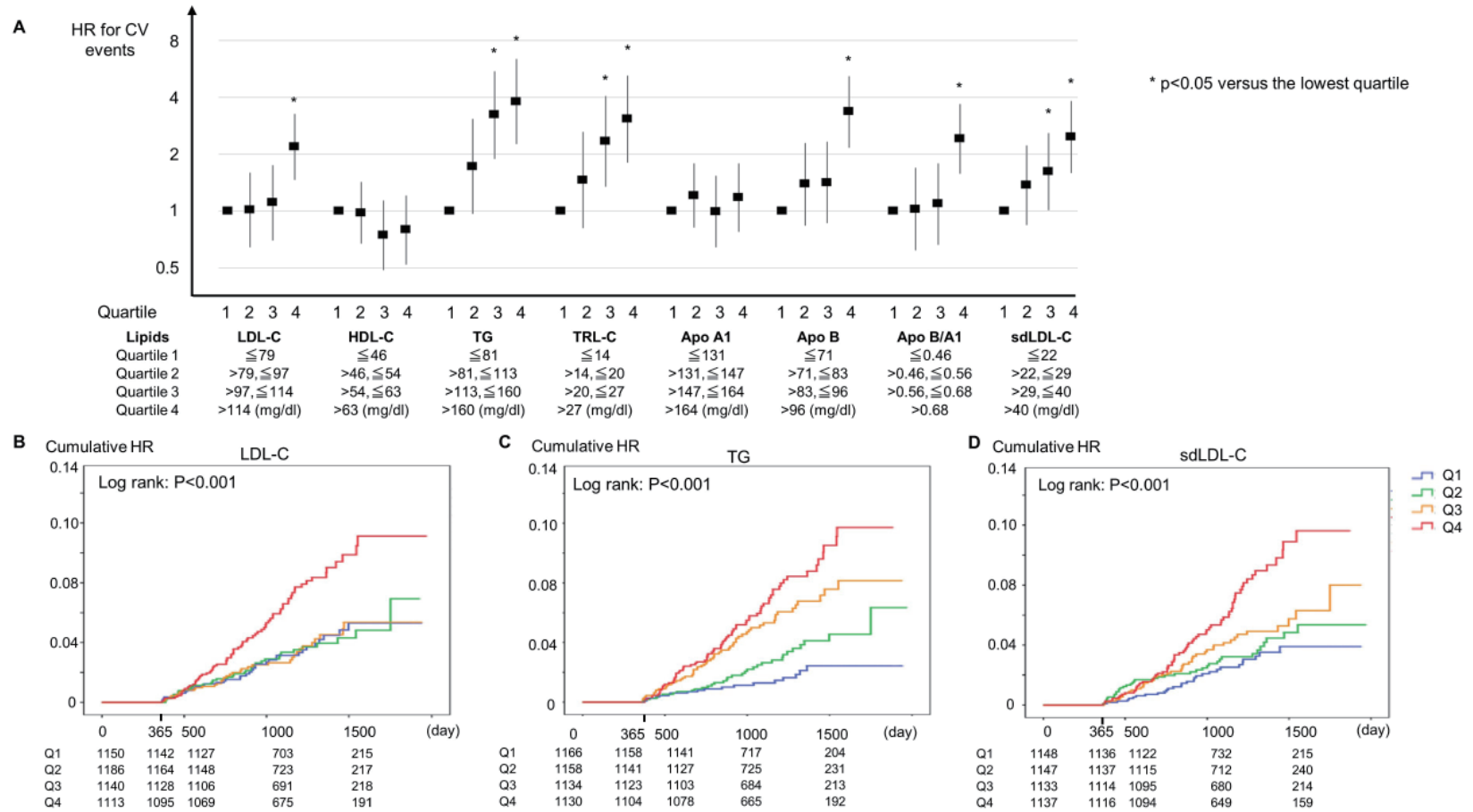

Fig.4. Hazard ratios of lipid levels for CV events

Hazard ratios for CV events comparing among the quartiles according to levels of each lipid are shown (A). Cumulative HR of LDL-C (B), TG (C), and sdLDL-C (D) for CV events were analyzed by Kaplan-Meier analysis. HR: Hazard ratios, LDL-C: low density lipoprotein cholesterol, HDL-C: high density lipoprotein cholesterol, TG: triglyceride, TRL-C: triglyceride-rich lipoprotein cholesterol, ApoA1: apolipoprotein A1, Apo B: apolipoprotein B, sdLDL-C: small dense low-density lipoprotein cholesterol, CV: cardiovascular.

A Cumulative hazard ratio of $\mathrm{CV}$ events

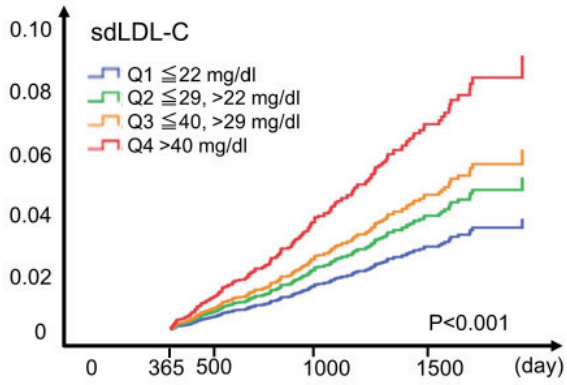

B Cumulative hazard ratio of the need for laser treatment

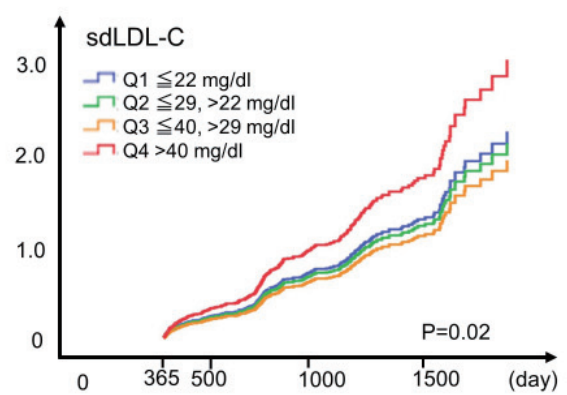

Fig. 5. Cumulative hazard ratios of lipid levels for CV events and the need for laser treatment for diabetic retinopathy

Cumulative hazard ratio of CV events and the need for laser treatment for diabetic retinopathy were analyzed by Cox hazard analysis after adjusting for covariates including age, gender, hypertension, and current or previous smoking at registration. LDL-C: low density lipoprotein cholesterol, sdLDL-C: small dense low-density lipoprotein cholesterol, CV: cardiovascular.

to levels of each lipid at one year after registration showed that TG, TRL-C, and sdLDL-C levels were positively related to the risk of $\mathrm{CV}$ events (TG: Q1, HR 1.0; Q2, HR 1.7; Q3, HR 3.2; Q4, HR 3.8, $p$ for trend <0.01. TRL-C: Q1, HR 1.0; Q2, HR 1.5; Q3, HR 2.3; Q4, HR 3.1, $p$ for trend <0.01. sdLDL-C: Q1, HR 1.0; Q2, HR 1.4; Q3, HR 1.6; Q4, HR 2.5, $p$ for trend <0.01) (Fig.4A). The Kaplan-Meier analysis showed a significantly higher risk for $\mathrm{CV}$ events as the levels of LDL-C, TG, and sdLDL-C at one year after registration are higher $(\log$ rank, $p<$ 0.001 for all) (Fig. 4B-D) and Cox hazard analysis showed a significantly higher risk for $\mathrm{CV}$ events according to the higher levels of sdLDL-C at one year after registration $(p<0.001)$ (Fig. 5A).

In patients with TG levels in the lower half $(\leqq$

\section{Advance Publication Journal of Atherosclerosis and Thrombosis}




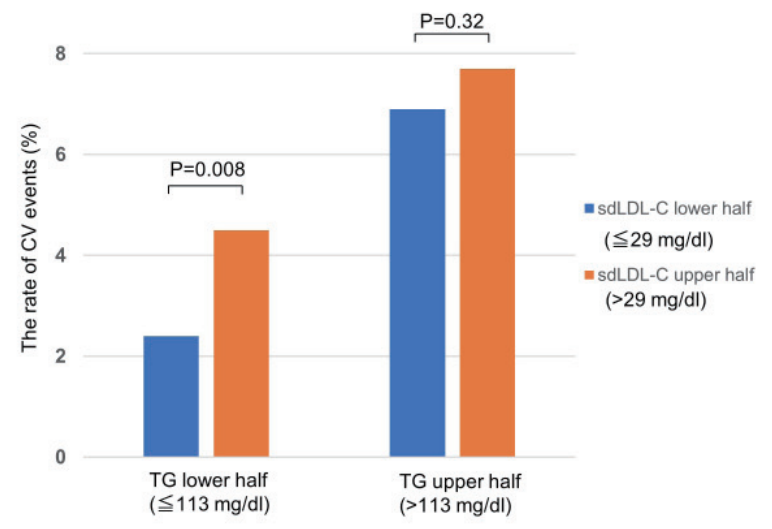

Fig. 6. The rate of CV events according to TG and sdLDL-C levels

The study population was divided into 2 subgroups, lower and upper halves of TG levels. The rates of CV events during the follow-up period were compared between the lower and upper halves of sdLDL-C levels in each subgroup by the chi squared test. CV: cardiovascular, TG: triglyceride, sdLDL-C: small dense low-density lipoprotein cholesterol.

A

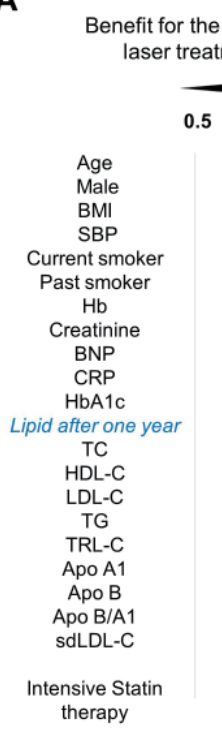

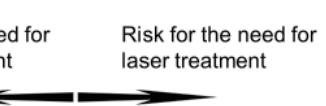

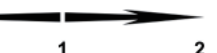

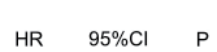

$0.96 \quad 0.89-1.03 \quad 0.23$

$\begin{array}{lll}1.11 & 0.95-1.31 & 0.19\end{array}$

$\begin{array}{lll}0.98 & 0.91-1.05 & 0.59\end{array}$

$\begin{array}{lll}1.02 & 0.95-1.10 & 0.60\end{array}$

$\begin{array}{lll}1.02 & 0.81-1.28 & 0.89\end{array}$

$\begin{array}{lll}1.02 & 0.82-1.27 & 0.85\end{array}$

$0.90 \quad 0.77-1.05 \quad 0.19$

$1.28 \quad 1.17-1.41<0.001$

$1.09 \quad 1.04-1.13<0.001$

$0.94 \quad 0.80-1.10 \quad 0.43$

$\begin{array}{lll}1.03 & 0.93-1.14 & 0.62\end{array}$

$\begin{array}{lll}1.03 & 0.94-1.14 & 0.49\end{array}$

$\begin{array}{llll}0.91 & 0.81-1.01 & 0.09\end{array}$

$1.09 \quad 0.99-1.20 \quad 0.09$

$\begin{array}{lll}1.09 & 1.00-1.19 & 0.11\end{array}$

$\begin{array}{lll}1.04 & 0.93-1.16 & 0.53\end{array}$

$\begin{array}{lll}1.04 & 0.93-1.16 & 0.53\end{array}$

$\begin{array}{lll}0.95 & 0.83-1.05 & 0.32 \\ 1.06 & 0.96-1.16 & 0.27\end{array}$

$\begin{array}{lll}1.06 & 0.96-1.16 & 0.27 \\ 1.09 & 0.96-1.24 & 0.17\end{array}$

$\begin{array}{lll}1.13 & 1.01-1.27 & 0.03\end{array}$

$\begin{array}{lll}1.18 & 0.96-1.44 \quad 0.12\end{array}$

\section{B}

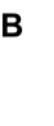

Age

Gender

Gender

BMI

Smoking

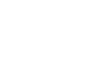

Hypertension

Creatinine

HbA1c (N)

HbA1c (N)

Diabetic
retinopathy retinopathy
status

status

Intensive Statin Yes
Sd LDL-C level is Not sensitive for predicting _ Sd LDL-C level is the need for laser treatment $\quad \begin{aligned} & \text { sensitive for predicting } \\ & \text { the need for laser treatment }\end{aligned}$

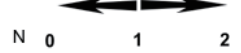

$\begin{array}{cccc}\text { HR } & 95 \% \mathrm{Cl} & \mathrm{P} & \begin{array}{c}\mathrm{P} \text { for } \\ \text { interaction }\end{array} \\ 1.18 & 1.03-1.34 & 0.02 & 0.11\end{array}$

$\begin{array}{lll}1.18 & 1.03-1.34 & 0.02\end{array}$ $0.95 \quad 0.82-1.10 \quad 0.50$

$\begin{array}{lll}1.01 & 0.88-1.15 & 0.92\end{array}$ $\begin{array}{lll}1.14 & 0.99-1.31 & 0.07\end{array}$

$\begin{array}{lll}1.17 & 1.02-1.33 & 0.02\end{array}$ $\begin{array}{lll}1.01 & 0.87-1.17 & 0.89\end{array}$

$\begin{array}{lll}1.07 & 0.82-1.40 & 0.61\end{array}$ $\begin{array}{lll}1.12 & 0.93-1.37 & 0.24\end{array}$ $\begin{array}{lll}1.08 & 0.95-1.22 & 0.26\end{array}$

$\begin{array}{lll}1.08 & 0.97-1.21 & 0.15\end{array}$ $\begin{array}{lll}1.04 & 0.85-1.28 & 0.71\end{array}$

$\begin{array}{lll}0.69 & 0.45-1.06 & 0.09\end{array}$ $\begin{array}{llll}1.08 & 0.98-1.20 & 0.12\end{array}$

$\begin{array}{lll}1.05 & 0.94-1.18 & 0.36\end{array}$ $\begin{array}{lll}1.15 & 0.94-1.42 & 0.17\end{array}$

$\begin{array}{lll}1.16 & 0.68-1.98 & 0.59\end{array}$

$\begin{array}{lll}1.16 & 0.68-1.98 & 0.59 \\ 1.06 & 0.90-1.24 & 0.50\end{array}$ $\begin{array}{lll}1.06 & 0.90-1.24 & 0.50 \\ 1.12 & 0.95-1.32 & 0.16\end{array}$ $\begin{array}{lll}1.12 & 0.95-1.32 & 0.16 \\ 1.14 & 0.90-1.43 & 0.29\end{array}$

$\begin{array}{lll}1.09 & 0.95-1.25 & 0.24\end{array}$ $\begin{array}{lll}1.99 & 0.95-1.25 & 0.24 \\ 0.85-1.16 & 0.93\end{array}$
0.20

0.03

0.67

0.57

0.22

0.19

0.13

0.14

Fig. 7. Hazard ratios for the need for laser treatment

Hazard ratios were estimated per one standard deviation increase by a Cox proportional analysis on the risks for the need for laser treatment after adjusting for covariates including age, gender, hypertension, and current or previous smoking at registration (A). Subgroup analysis (B). $\mathrm{Hb}$ : hemoglobin, BNP: brain natriuretic peptide, CRP: C-reactive protein, TC: total cholesterol, HDL-C: high density lipoprotein cholesterol, LDL-C: low density lipoprotein cholesterol, TG: triglyceride, TRL-C: triglyceride-rich lipoprotein cholesterol, ApoA1: apolipoprotein A1, Apo B: apolipoprotein B, sdLDL-C: small dense low-density lipoprotein cholesterol.

$113 \mathrm{mg} / \mathrm{dl})$, those with sdLDL-C levels in the upper half $(>29 \mathrm{mg} / \mathrm{dl})$ had more CV events than those with sdLDL-C in the lower half $(\leqq 29 \mathrm{mg} / \mathrm{dl})(\mathrm{CV}$ events rate: 4.5 vs. $2.4 \%$, respectively, $p<0.01$ ) (Fig. 6), and the sdLDL-C $(>29 \mathrm{mg} / \mathrm{dl})$ was a significant marker for predicting CV events (HR 1.86, $95 \%$ CI 1.16-3.00, $p=0.01)$. In patients with TG levels in the upper half $(>113 \mathrm{mg} / \mathrm{dl})$, sdLDL-C (> $29 \mathrm{mg} / \mathrm{dl}$ ) was not a significant marker for predicting
CV events (HR 1.09, 95\% CI 0.77-1.54, $p=0.63$ ).

\section{Laser Treatment for Diabetic Retinopathy}

In Cox proportional hazard analysis, higher levels of creatinine, BNP and sdLDL -C were risks of the need for laser treatment (HR 1.28, 95\% CI 1.17-1.41; HR 1.09, 95\% CI 1.04-1.13; HR 1.13, 95\% CI 1.011.27, respectively) (Fig.7A). The Kaplan-Meier analysis and Cox hazard analysis showed a significantly

\section{Advance Publication Journal of Atherosclerosis and Thrombosis}



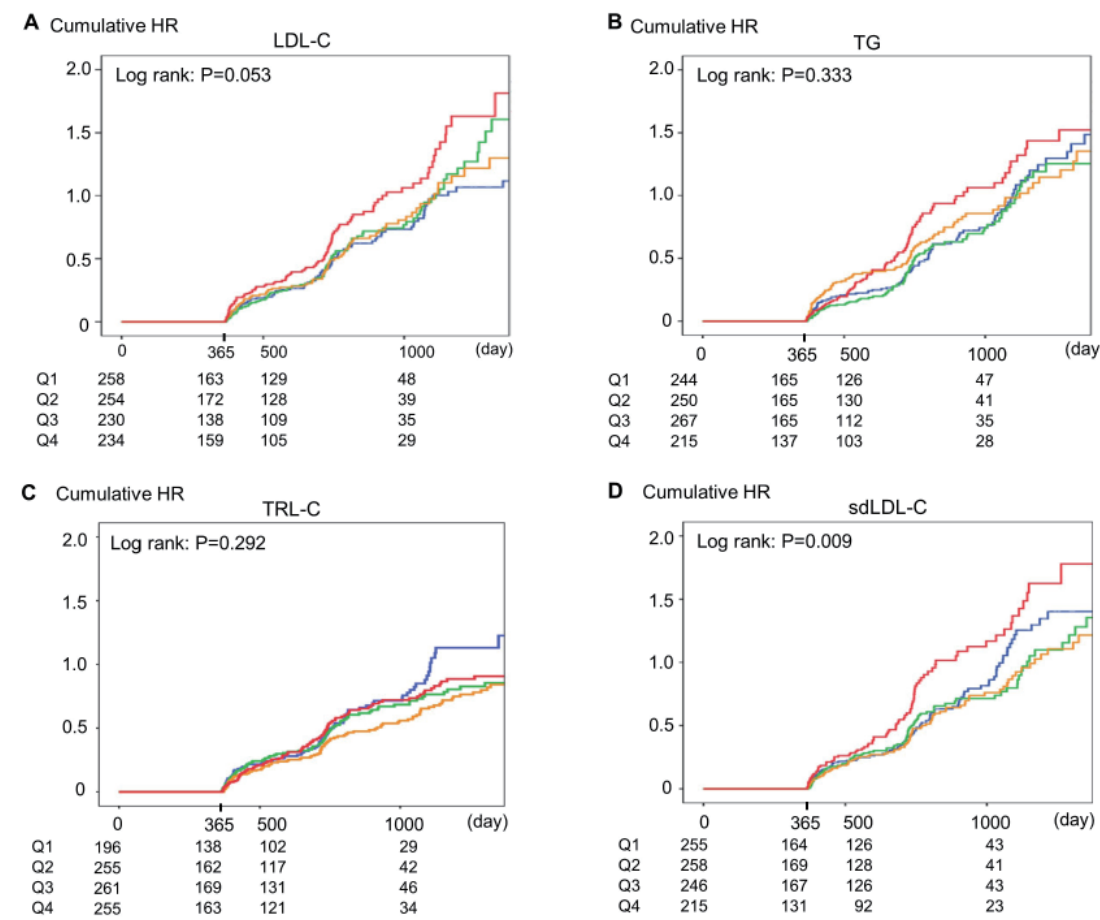

Fig. 8. Cumulative hazard ratios of lipid values for the need for laser treatment for diabetic retinopathy

Cumulative HR of LDL-C (A), TG (B), TRL-C (C) and sdLDL-C (D) for the need for laser treatment for diabetic retinopathy were analyzed by Kaplan-Meier analysis. HR: Hazard ratios, LDL-C: low density lipoprotein cholesterol, TG: triglyceride, TRL-C: triglyceride-rich lipoprotein cholesterol, sdLDL-C: small dense low-density lipoprotein cholesterol.

higher risk of laser treatment as the levels of sdLDL-C at one year after registration are higher (log rank, $p=0.009$, and $p=0.02$ ) (Fig. 8D, Fig. 5B), and HR of the need for laser treatment in the highest quartile group $(>40 \mathrm{mg} / \mathrm{dl})$ of sdLDL-C as compared with the lowest quartile group ( $\leqq 22 \mathrm{mg} / \mathrm{dl})$ was $1.35(95 \%$ CI 1.01-1.80) (Fig.5B). No other lipid except for sdLDL-C was significantly associated with the need for laser treatment (Fig.7A, Fig. 8A-C). Particular in the sub-populations of older age ( $\geqq 65$ years) and obesity (BMI $\geqq 25 \mathrm{~kg} / \mathrm{m}^{2}$ ), sdLDL-C was significantly associated with the need for laser treatment for diabetic retinopathy (HR 1.18, 95\% CI 1.03-1.34; HR 1.17, 95\% CI 1.02-1.33, respectively) (Fig. 7B).

There was no significant correlation between sdLDL-C and urine albumin or eGFR. After adjustment for the existence of nephropathy, the sdLDL-C level remained to be a risk of the need for laser treatment (HR 1.12, 95\% CI 1.01-1.23), suggesting that nephropathy and the sdLDL-C level exert as independent risks of the need for laser treatment.

\section{Discussion}

This study clarified that TC, LDL-C, TG, TRL-
C, ApoB and sdLDL-C were lipid markers to predict $\mathrm{CV}$ events, depending on their concentrations, and sdLDL-C was the only lipid marker to predict the need for laser treatment in patients with hypercholesterolemia and diabetic retinopathy receiving statin therapy. To our best knowledge, this is the largest study so far to examine prospectively and comprehensively which lipid marker could predict CV events and the need for laser treatment in diabetic patients receiving statin therapy.

\section{Correlations among Lipids}

SdLDL is a particle with a specific density of $1.044-1.063 \mathrm{~g} / \mathrm{ml}$ and a size of $19.0-20.5 \mathrm{~nm}^{9,18)}$. As compared with lbLDL, sdLDL particles have lower affinity to the LDL receptor, and longer retention time in the circulation. In addition, sdLDL easily adheres to proteoglycans in the vessel wall, and easily penetrates the subendothelium of blood vessels (Fig.9) ${ }^{19)}$. Compared with other LDL particles, sdLDL particles are more susceptible to oxidative modification and more readily engulfed by macrophages leading to greater levels of oxidized-LDL particles as well as vascular damage (Fig.9). Consistently, elevated sdLDL-C values correspond to development of unstable and rupture-prone plaque 


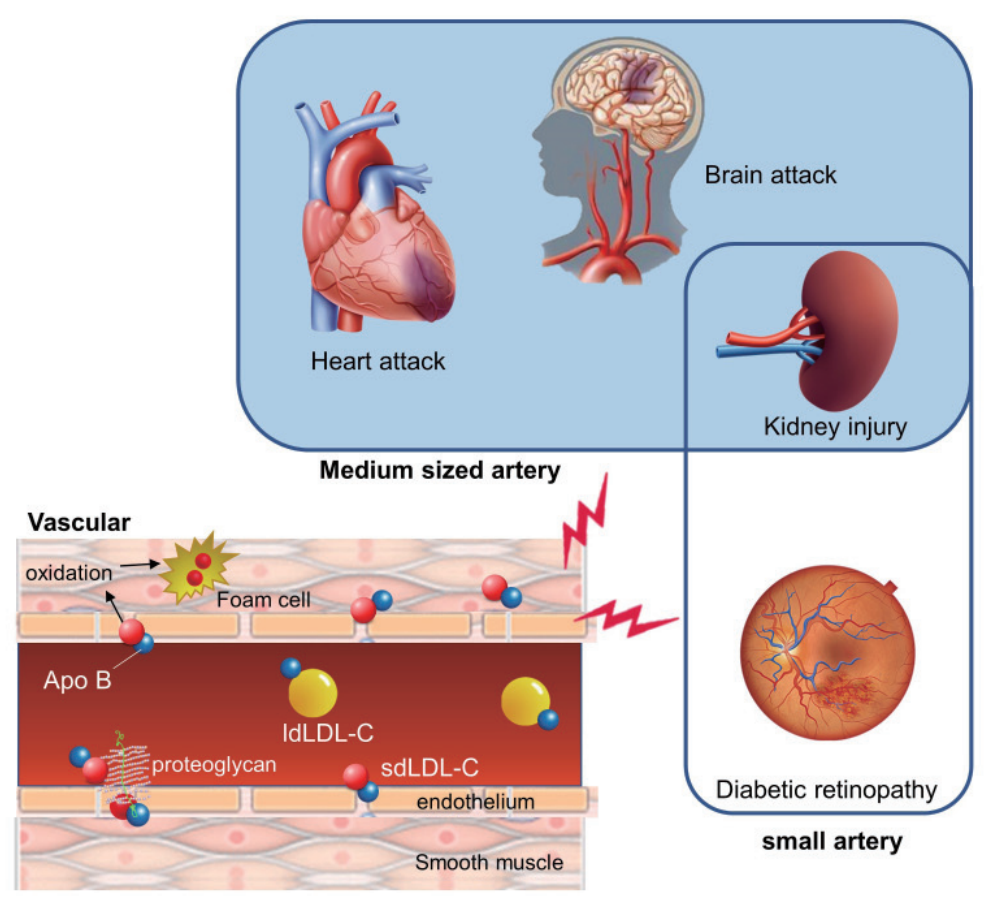

Fig. 9. The role of small dense low-density lipoprotein cholesterol

SdLDL-C easily adheres to proteoglycans in the vessel wall and easily penetrates the subendothelium of blood vessels, leading to greater levels of oxidized-LDL particles as well as vascular damage in both medium- and small-sized arteries. Apo B: apolipoprotein B, lbLDL-C: large buoyant low-density lipoprotein cholesterol, sdLDL-C: small dense low-density lipoprotein cholesterol.

phenotypes relevant to CV events.

Overproduction of Apo B-containing particles by the liver leads to the excessive biosynthesis of the heavier subfraction of LDL. ApoB reflects LDL particle number because all lipoprotein particles contain one molecule of $A p o B^{18)}$, and sdLDL is the most numerous particle among LDL particles ${ }^{20)}$. Accordingly, there should be the close correlation between sdLDL-C and ApoB levels. A high correlation between sdLDL-C and ApoB values in small study populations was reported ${ }^{21,22}$. Consistent with these findings, in the present study $(n=4589)$, sdLDL-C level was strongly correlated with ApoB level $(\mathrm{r}=0.83$, $p<0.001)$.

\section{Lipid Markers for Predicting CV Events}

In the population from the EMPATHY study, we explored the association between lipid values and the future onset of CV events. TC, LDL-C, TG, TRL$\mathrm{C}, \mathrm{ApoB}$ and sdLDL-C at one year after registration were significantly associated with the risk of $\mathrm{CV}$ events. Usefulness of measuring TG for predicting the $\mathrm{CV}$ events was previously reported in many studies including the EMPATHY study ${ }^{23,24)}$. Moreover, it was shown that non-fasting TG could be considered to be a substitute for fasting TG as a risk-stratification for future $\mathrm{CV}$ events ${ }^{23)}$. The Copenhagen City Heart
Study showed that the non-fasting TG level was correlated with the risks of ischemic heart diseases and stroke ${ }^{25}$. The European Atherosclerosis Society and the European Society of Laboratory Medicine jointly published the statement that postprandial samples may be used routinely to assess lipid profiles, and suggested their usefulness and convenience in daily clinical practice ${ }^{26)}$. Despite these arguments, the clinical usefulness of the measurement of non-fasting TG seems still controversial ${ }^{27)}$ and we prefer the biomarker which is less susceptible to dietary influence. In this regard, sdLDL-C, which is much less susceptible to dietary influences ${ }^{28)}$, might be a better lipid marker measured in daily clinical practice as compared with TGs.

TRL-C, which contains very low-density lipoproteins, intermediate-density lipoproteins, chylomicrons, and their remnants, has been recently paid attention as the substrate causally related to development of the atherosclerotic plaque ${ }^{5)}$. In our study, TRL-C was reduced by statin therapy and exerted as the significant marker for predicting $\mathrm{CV}$ events, but not for predicting the need of laser treatment.

In Framingham Heart Study, women with CAD had higher sdLDL-C values as compared with controls $^{29)}$. Sd-LDL-C values were most closely 
correlated with carotid artery intima-media thickness among lipid parameters tested ${ }^{30}$. In the recent epidemiological studies, sdLDL-C values are associated with increased risk of $\mathrm{CV}$ events ${ }^{8,31)}$ and incident myocardial infarction ${ }^{32}$. Consistently, in our large-scale prospective study of diabetic patients, we could clearly demonstrate that sdLDL-C is the good predictor of incident $\mathrm{CV}$ events among lipid parameters.

Lipid Markers for Predicting the Need for Laser Treatment

The association between lipids and risk for worsening diabetic retinopathy or the need for laser treatment is controversial ${ }^{33)}$. In 1998, Davis et al. demonstrated that elevated TG, but not TC, HDL-C, or LDL-C, was significantly associated with high-risk proliferative diabetic retinopathy in diabetic patients ${ }^{34)}$. The recent Mendelian randomization study in Copenhagen cohorts found that elevated LDL-C value was not associated with risk of retinopathy and showed that LDL-C had no causal relationship with microvascular diseases such as retinopathy ${ }^{35)}$. The FIELD study ${ }^{12)}$ showed HDL-C, LDL-C, and TG levels were not the significant markers for laser treatment. However, fibrate ${ }^{12)}$ and statin $^{36)}$ intake was shown to reduce the need for laser treatment in randomized controlled trial and metaanalysis. Taken together, there might be a weak or borderline association between LDL-C and the risk for diabetic retinopathy. LDL-C complex, which consists of lbLDL-C, sdLDL-C, and other subfractions, could not serve as a robust marker for worsening diabetic retinopathy. Theoretically, sdLDL-C could easily flow inside the retinal artery walls, augmenting the oxidative stress, followed by the advanced proliferative change in retina. As far as we know, this is the first study to demonstrate that elevated sdLDL-C level is significantly associated with increased risk of the need for laser treatment for diabetic retinopathy. Hereafter, further clinical studies are warranted to clarify whether sdLDL-C lowering therapy could have a suppressive effect on the progression of diabetic retinopathy as well as $\mathrm{CV}$ diseases.

\section{Clinical Implications for the Measurement of sdLDL-C Values}

Considering that higher TG values are associated with smaller LDL particle sizes, TG values should be associated with sdLDL-C values ${ }^{22)}$. However, in the present study, a high correlation between TG and sdLDL-C was not observed $(r=0.58)$. Rather, even in patients with TG levels in the lower half, sdLDL-C was a significant marker for predicting CV events. Given that, sdLDL-C was a sensitive marker to predict future $\mathrm{CV}$ events, independent of $\mathrm{TG}$ level. In addition, sdLDL-C was found to be the only lipid marker to predict the need for laser treatment. Although ApoB level was strongly correlated with sdLDL-C level $(\mathrm{r}=0.83, p<0.001)$, ApoB level was not associated with the need for laser treatment in our study. ApoB is a component of a variety of lipoproteins (eg. lb LDL-C ${ }^{21)}$ ) whose pathogenic properties in the vascular wall might vary depending on vascular size, which could partly explain the reason why ApoB itself could not predict the prognosis of retinopathy. Taken together, sdLDL-C levels should be evaluated as a predictive maker for future $\mathrm{CV}$ events and the need for laser treatment in patients at risk for $\mathrm{CV}$ events and diabetic retinopathy.

\section{Limitations}

This study was a sub-analysis of the EMPATHY study ${ }^{13)}$. This large-scale PROBE study design could minimize the effect of confounding factors. However, study participants were limited to patients with hypercholesterolemia and diabetic retinopathy receiving statin therapy; therefore, our findings cannot be simply generalized to the other populations. Blood sampling was performed at each clinic in the EMPATHY study ${ }^{13)}$, and we cannot deny the possibility of postprandial changes in lipid profile, especially TG. Finally, after the exclusion of patients with history of treatment for retinopathy including laser treatment or without routine ophthalmologic check-up until 3 years after registration, it was the remaining 976 patients that we used for the analysis on the need for laser treatment. Also, in this study, the need for laser treatment for diabetic retinopathy was decided by the ophthalmologists in each institution, which could have biased the ophthalmologic endpoint. Further studies with larger sample size are required to confirm the significance of sd LDL-C levels in this field.

\section{Conclusions}

SdLDL-C can serve as a sensitive target marker to predict both the occurrence of cardiovascular events and the need for laser treatment in patients with hypercholesterolemia and diabetic retinopathy. SdLDL-C levels should be evaluated in patients at risk for cardiovascular events as well as diabetic retinopathy. 


\section{Data Availability}

The data that support the findings of this study are available from the EMPATHY data center, but restrictions apply to the availability of these data, which were used under license for the current study, and so are not publicly available. Data are available from the authors upon reasonable request and with permission of the EMPATHY Investigators.

\section{Disclosures / Conflicts of Interest}

H.I. reports grants and personal fees from Shionogi \& Co., Ltd during the course of the study, and grants and personal fees from Takeda Pharmaceutical Co. Ltd, Nippon Boehringer Ingelheim Co., Ltd, Daiichi Sankyo Co., Ltd, MSD K.K., Mitsubishi Tanabe Pharma Corporation, Shionogi \& Co., Ltd and Taisho Toyama Pharmaceutical Co., Ltd, as well as grants from Sumitomo Dainippon Pharma Co., Ltd, Astellas Pharma Inc., Kyowa Hakko Kirin Co., Ltd, Teijin Pharma Ltd, Mochida Pharmaceutical Co., Ltd, Ono Pharmaceutical Co., Ltd, Chugai Pharmaceutical Co., Ltd, Eli Lilly Japan K.K. and personal fees from Nipro Corporation and SBI Pharmaceuticals Co., Ltd outside the submitted work. I.K. reports personal fees from Shionogi \& Co., Ltd during the course of the study, grants and personal fees from Takeda Pharmaceutical Co. Ltd, Nippon Boehringer Ingelheim Co., Ltd, Astellas Pharma Inc., Daiichi Sankyo Co., Ltd, and Otsuka Pharmaceutical Co., Ltd and grants from MSD K.K., Shionogi \& Co., Ltd, GlaxoSmithKline K.K., Sanofi K.K., Genzyme Japan K.K., Sumitomo Dainippon Pharma Co., Ltd, Mitsubishi Tanabe Pharma Corporation and Bristol Myers Squibb Co. outside the submitted work.

\section{Notice of Grant Support}

None.

\section{Funding}

None.

\section{Authors' Contributions}

AN and TS contributed to the study design. AN and TK contributed to the statistical plan. AN and TK analyzed the data and prepared the study results. HM contributed to the interpretation of the findings. NT, SK, HI, and IK supervised the study project. AN led the drafting of the manuscript, and all co-authors contributed to revising of the manuscript and approved the final version.

\section{Acknowledgements}

None.

\section{References}

1) Ingelsson E, Schaefer EJ, Contois JH, McNamara JR, Sullivan L, Keyes MJ, Pencina MJ, Schoonmaker C, Wilson PW, D'Agostino RB, Vasan RS. Clinical utility of different lipid measures for prediction of coronary heart disease in men and women. JAMA, 2007; 298: 776-785

2) McQueen MJ, Hawken S, Wang $X$, Ounpuu $S$, Sniderman A, Probstfield J, Steyn K, Sanderson JE, Hasani M, Volkova E, Kazmi K, Yusuf S; INTERHEART study investigators. Lipids, lipoproteins, and apolipoproteins as risk markers of myocardial infarction in 52 countries (the INTERHEART study): a casecontrol study. Lancet, 2008; 372: 224-233

3) Catapano AL, Graham I, De Backer G, Wiklund O, Chapman MJ, Drexel $\mathrm{H}$, Hoes AW, Jennings CS, Landmesser U, Pedersen TR, Reiner Ž, Riccardi G, Taskinen MR, Tokgozoglu L, Verschuren WMM, Vlachopoulos C, Wood DA, Zamorano JL, Cooney MT; ESC Scientific Document Group. 2016 ESC/EAS Guidelines for the Management of Dyslipidaemias. Eur Heart J, 2016; 37: 2999-3058

4) Goldberg RB, Stone NJ, Grundy SM. The $2018 \mathrm{AHA} /$ ACC/AACVPR/AAPA/ABC/ACPM/ADA/AGS/APhA/ ASPC/NLA/PCNA Guidelines on the Management of Blood Cholesterol in Diabetes. Diabetes Care, 2020; 43: $1673-1678$

5) Vallejo-Vaz AJ, Fayyad R, Boekholdt SM, Hovingh GK, Kastelein JJ, Melamed S, Barter P, Waters DD, Ray KK. Triglyceride-Rich Lipoprotein Cholesterol and Risk of Cardiovascular Events Among Patients Receiving Statin Therapy in the TNT Trial. Circulation, 2018; 138: 770781

6) Gianfagna F, Veronesi G, Guasti L, Chambless LE, Brambilla P, Corrao G, Mancia G, Cesana G, Ferrario MM. Do apolipoproteins improve coronary risk prediction in subjects with metabolic syndrome? Insights from the North Italian Brianza cohort study. Atherosclerosis, 2014; 236: 175-181

7) Walldius G, Jungner I. Is there a better marker of cardiovascular risk than LDL cholesterol? Apolipoproteins B and A-I--new risk factors and targets for therapy. Nutr Metab Cardiovasc Dis, 2007; 17: 565-571

8) Hoogeveen RC, Gaubatz JW, Sun W, Dodge RC, Crosby JR, Jiang J, Couper D, Virani SS, Kathiresan S, Boerwinkle E, Ballantyne CM. Small dense low-density lipoprotein-cholesterol concentrations predict risk for coronary heart disease: the Atherosclerosis Risk In Communities (ARIC) study. Arterioscler Thromb Vasc Biol, 2014; 34: 1069-1077

9) Talebi S, Bagherniya M, Atkin SL, Askari G, Orafai HM, Sahebkar A. The beneficial effects of nutraceuticals and natural products on small dense LDL levels, LDL particle 
number and LDL particle size: a clinical review. Lipids Health Dis, 2020; 19: 66

10) Leasher JL, Bourne RR, Flaxman SR, Jonas JB, Keeffe J, Naidoo K, Pesudovs K, Price H, White RA, Wong TY, Resnikoff S, Taylor HR; Vision Loss Expert Group of the Global Burden of Disease Study. Global Estimates on the Number of People Blind or Visually Impaired by Diabetic Retinopathy: A Meta-analysis From 1990 to 2010. Diabetes Care, 2016; 39: 1643-1649

11) Frederick L. Ferris III. How Effective Are Treatments for Diabetic Retinopathy? JAMA, 1993; 269: 1290-1291

12) Keech A, Simes RJ, Barter P, Best J, Scott R, Taskinen MR, Forder P, Pillai A, Davis T, Glasziou P, Drury P, Kesäniemi YA, Sullivan D, Hunt D, Colman P, d'Emden M, Whiting M, Ehnholm C, Laakso M; FIELD study investigators. Effects of long-term fenofibrate therapy on cardiovascular events in 9795 people with type 2 diabetes mellitus (the FIELD study): randomised controlled trial. Lancet, 2005; 366: 1849-1861

13) Itoh $H$, Komuro I, Takeuchi $M$, Akasaka T, Daida $H$, Egashira Y, Fujita H, Higaki J, Hirata KI, Ishibashi S, Isshiki T, Ito $S$, Kashiwagi A, Kato S, Kitagawa $\mathrm{K}$, Kitakaze M, Kitazono T, Kurabayashi M, Miyauchi K, Murakami T, Murohara T, Node K, Ogawa S, Saito Y, Seino Y, Shigeeda T, Shindo S, Sugawara M, Sugiyama S, Terauchi Y, Tsutsui $\mathrm{H}$, Ueshima K, Utsunomiya K, Yamagishi M, Yamazaki T, Yo S, Yokote K, Yoshida K, Yoshimura M, Yoshimura N, Nakao K, Nagai R; EMPATHY Investigators. Intensive Treat-to-Target Statin Therapy in High-Risk Japanese Patients With Hypercholesterolemia and Diabetic Retinopathy: Report of a Randomized Study. Diabetes Care, 2018; 41: 12751284

14) Wong TY, Sun J, Kawasaki R, Ruamviboonsuk P, Gupta N, Lansingh VC, Maia M, Mathenge W, Moreker S, Muqit MMK, Resnikoff S, Verdaguer J, Zhao P, Ferris F, Aiello LP, Taylor HR. Guidelines on Diabetic Eye Care: The International Council of Ophthalmology Recommendations for Screening, Follow-up, Referral, and Treatment Based on Resource Settings. Ophthalmology, 2018; 125: 1608-1622

15) Grading diabetic retinopathy from stereoscopic color fundus photographs — an extension of the modified Airlie House classification. ETDRS report number 10. Early Treatment Diabetic Retinopathy Study Research Group. Ophthalmology, 1991; 98: 786-806

16) David H, Yann DR, Guillaume C, Florence T. Estimation of Conditional and Marginal Odds Ratios Using the Prognostic Score. Stat Med, 2017; 36: 687-716

17) Palazhy S, Viswanathan V. Lipid Abnormalities in Type 2 Diabetes Mellitus Patients with Overt Nephropathy. Diabetes Metab J, 2017; 41: 128-134

18) Ivanova EA, Myasoedova VA, Melnichenko AA, Grechko AV, Orekhov AN. Small Dense Low-Density Lipoprotein as Biomarker for Atherosclerotic Diseases. Oxid Med Cell Longev, 2017; 2017: 1273042

19) Elovson J, Chatterton JE, Bell GT, Schumaker VN, Reuben MA, Puppione DL, Reeve JR Jr, Young NL. Plasma very low density lipoproteins contain a single molecule of apolipoprotein B. J Lipid Res, 1988; 29: 1461-1473
20) Fernández-Cidón B, Candás-Estébanez B, Ribalta J, Rock E, Guardiola-Guionnet M, Amigó N, Padró-Miquel A, Alía-Ramos P, Pintó-Sala X. Precipitated sdLDL: An easy method to estimate LDL particle size. J Clin Lab Anal, 2020; 34: e23282

21) Hirano T, Ito $Y$, Saegusa H, Yoshino G. A novel and simple method for quantification of small, dense LDL. J Lipid Res, 2003; 44: 2193-2201

22) Nishikura T, Koba S, Yokota $Y$, Hirano T, Tsunoda F, Shoji M, Hamazaki Y, Suzuki H, Itoh Y, Katagiri T, Kobayashi Y. Elevated small dense low-density lipoprotein cholesterol as a predictor for future cardiovascular events in patients with stable coronary artery disease. J Atheroscler Thromb, 2014; 21: 755-767

23) Tada $H$, Nomura A, Yoshimura K, Itoh $H$, Komuro I, Yamagishi M, Takamura M, Kawashiri MA. Fasting and Non-Fasting Triglycerides and Risk of Cardiovascular Events in Diabetic Patients Under Statin Therapy. Circ J, 2020; 84: 509-515

24) Tada $H$, Kawashiri MA, Nomura A, Yoshimura K, Itoh $H$, Komuro I, Yamagishi M. Serum triglycerides predict first cardiovascular events in diabetic patients with hypercholesterolemia and retinopathy. Eur J Prev Cardiol, 2018; 25: 1852-1860

25) Jackson KG, Poppitt SD, Minihane AM. Postprandial lipemia and cardiovascular disease risk: Interrelationships between dietary, physiological and genetic determinants. Atherosclerosis, 2012; 220: 22-33

26) Nordestgaard BG, Langsted A, Mora S, Kolovou G, Baum H, Bruckert E, Watts GF, Sypniewska G, Wiklund O, Borén J, Chapman MJ, Cobbaert C, Descamps OS, von Eckardstein A, Kamstrup PR, Pulkki K, Kronenberg F, Remaley AT, Rifai N, Ros E, Langlois M; European Atherosclerosis Society (EAS) and the European Federation of Clinical Chemistry and Laboratory Medicine (EFLM) joint consensus initiative. Fasting is not routinely required for determination of a lipid profile: clinical and laboratory implications including flagging at desirable concentration cut-points-a joint consensus statement from the European Atherosclerosis Society and European Federation of Clinical Chemistry and Laboratory Medicine. Eur Heart J, 2016; 37: 1944-1958

27) Fischer S, Schatz U, Julius U. Practical recommendations for the management of hyperlipidemia. Atheroscler Suppl, 2015; 18: 194-198

28) Farukhi ZM, Demler OV, Caulfield MP, Kulkarni K, Wohlgemuth J, Cobble M, Luttmann-Gibson H, Li C, Nelson JR, Cook NR, Buring JE, Krauss RM, Manson JE, Mora S. Comparison of nonfasting and fasting lipoprotein subfractions and size in 15,397 apparently healthy individuals: An analysis from the VITamin D and OmegA-3 TriaL. J Clin Lipidol, 2020; 14: 241-251

29) Ai M, Otokozawa S, Asztalos BF, Ito $Y$, Nakajima $K$, White CC, Cupples LA, Wilson PW, Schaefer EJ. Small dense LDL cholesterol and coronary heart disease: results from the Framingham Offspring Study. Clin Chem, 2010; 56: 967-976nary heart disease: results from the Framingham Offspring Study. Clin Chem, 2010; 56: 967-976

30) Shoji T, Hatsuda $S$, Tsuchikura $S$, Shinohara K, Kimoto E, Koyama H, Emoto M, Nishizawa Y. Small dense low- 
density lipoprotein cholesterol concentration and carotid atherosclerosis. Atherosclerosis, 2009; 202: 582-588

31) Arai H, Kokubo Y, Watanabe M, Sawamura T, Ito $Y$, Minagawa A, Okamura T, Miyamato Y. Small dense lowdensity lipoproteins cholesterol can predict incident cardiovascular disease in an urban Japanese cohort: the Suita study. J Atheroscler Thromb, 2013; 20: 195-203

32) Duran EK, Aday AW, Cook NR, Buring JE, Ridker PM, Pradhan AD. Triglyceride-Rich Lipoprotein Cholesterol, Small Dense LDL Cholesterol, and Incident Cardiovascular Disease. J Am Coll Cardiol, 2020; 75: 2122-2135

33) Chang YC, Wu WC. Dyslipidemia and diabetic retinopathy. Rev Diabet Stud, 2013; 10: 121-132

34) Davis MD, Fisher MR, Gangnon RE, Barton F, Aiello
LM, Chew EY, Ferris FL 3rd, Knatterud GL. Risk factors for high-risk proliferative diabetic retinopathy and severe visual loss: Early Treatment Diabetic Retinopathy Study Report \#18. Invest Ophthalmol Vis Sci, 1998; 39: 233252

35) Emanuelsson F, Nordestgaard BG, Tybjærg-Hansen A, Benn M. Impact of LDL Cholesterol on Microvascular Versus Macrovascular Disease: A Mendelian Randomization Study. J Am Coll Cardiol, 2019; 74: 1465-1476

36) Pranata R, Vania R, Victor AA. Statin reduces the incidence of diabetic retinopathy and its need for intervention: A systematic review and meta-analysis. Eur J Ophthalmol. 2020 Jun 12: 1120672120922444 . Eur J Ophthalmol, 2020; 1120672120922444 